Introduction: There are insufficient data on surface mold brachytherapy (SMB) in treating oral cancers. We reviewed our institutional experience to investigate the efficacy and toxicity of this treatment modality.

Material and methods: We retrospectively reviewed all the patients treated between 1989 and 2018 with high-dose-rate iridium-192 SMB for oral and oropharyngeal squamous cell carcinomas at our institution. Surface mold brachytherapy was delivered via an acrylic surface mold with 1-5 inserted catheters spaced $1 \mathrm{~cm}$ apart fabricated by our dental oncologist. The Kaplan-Meier product estimator was used to assess local control (LC), locoregional control (LRC), distant metastasis-free survival (DMFS), and overall survival (OS). Cox proportional hazards regression analysis was used to assess the relationship of various variables and patient outcomes.

Results: Eighteen patients met the inclusion criteria and were evaluated. Indications for treatment were primary tumor $(n=13)$, local recurrence (2), locoregional recurrence (1), and oligometastatic disease (1). Ten patients received SMB alone and 8 received external beam radiotherapy with an SMB boost. The acute toxicity outcomes were as follows: no toxicity $(n=1)$, grade 1 (7), grade $2(9)$, and grade 3 (1). Late effects were rare, only occurring in 3 patients. The one- and two-year LC were $81 \%$ and $68 \%$, LRC $77 \%$ and $64 \%$, DMFS $81 \%$ and $81 \%$, and OS $77 \%$ and $46 \%$.

Conclusions: Surface mold brachytherapy is a viable modality as either primary or boost treatment for superficial oral cancers. In our patients, this treatment method has a low toxicity profile and resulted in reasonable LC.

Key words: surface mold brachytherapy, head and neck cancer, brachytherapy, oral cancer, oropharyngeal cancer, head and neck cancer.

Contemp Oncol (Pozn) 2021; 25 (4): 254-263 DOI: https://doi.org/10.5114/wo.2021.111087

\section{Surface mould brachytherapy in oral and oropharyngeal cancers}

\author{
Leonid B. Reshko, Jeremy T. Gaskins, Jeffrey M. Bumpous, \\ Paul A. Tennant, Zafrulla Khan, Keith Sowards, Craig L. Silverman, \\ Neal E. Dunlap
}

University of Louisville, the United States of America

\section{Introduction}

Oral or oropharyngeal squamous cell carcinomas are a major source of morbidity and mortality [1-3]. Treatment of these cancers depends on the stage and risk factors, generally including definitive radiotherapy with or without chemotherapy or surgery followed by risk-adapted adjuvant therapy [4]. External beam radiotherapy (EBRT) is the most common type of radiotherapy for oral and oropharyngeal cancers when radiation therapy is indicated [4,5]. External beam radiation therapy is known to cause significant toxicity including mucositis, xerostomia, odynophagia, fibrosis, fistula formation, dental problems, osteoradionecrosis, and trismus. Brachytherapy can be used to reduce the radiation dose to the normal structures. Two forms of brachytherapy are available: interstitial brachytherapy for deeply invasive tumors and surface mold brachytherapy (SMB) for superficial cancers. In select cases, major salivary glands and skin can be spared by using these techniques. Most of the studies published on oral cancer brachytherapy used an interstitial technique [6-9]. Local control (LC) rates of $60-90 \%$ have been achieved [10-12]. The placement of these catheters is an invasive procedure that requires general anesthesia and special expertise. Brachytherapy has been shown in retrospective studies to provide comparable survival, LC, and toxicity profile to historical controls with EBRT $[10,13]$.

Unlike interstitial brachytherapy, SMB does not require an invasive procedure. It is a well-tolerated outpatient technique. Surface mold brachytherapy has been shown in retrospective studies to provide acceptable survival and LC with reasonable toxicity outcomes. While several retrospective reports have been published, most were done in Asia [3, 7, 14-17] and often included skin cancers [15]. Due to the limitations of these retrospective reports, particularly their very small sample size and a high degree of heterogeneity and absence of prospective data, additional studies are needed [3, 7, 14, 15-17]. We report our institutional experience with SMB in an American group of oral and oropharyngeal cancer patients. Disease-specific outcomes and toxicity were evaluated.

\section{Material and methods}

\section{Patients}

We performed a retrospective review of all patients treated consecutively at our institution between 1989 and 2018 with high-dose-rate iridium-192 (Ir-192) SMB for superficial oral and oropharyngeal malignancies. These were defined as tumors that would be completely encompassed by the brachytherapy prescription depth of $0.5-1 \mathrm{~cm}$. The goal of treatment was primarily curative, but patients treated for durable LC in an oligometastatic disease setting were included as well. In all cases, our institutional multidisciplinary tumor board including radiation oncologists, head and neck surgeons, medical oncologists, dental oncologists, diagnostic radiologists, and pathologists discussed the cases and recommended a treatment approach. 
Patients were generally followed every 3-6 months for the first two years and then 6-12 months subsequently after the completion of brachytherapy. Detailed electronic medical records were complete and available for all patients included in the study. Treatment-related toxicity was classified through CTCAE version 5 [18]. Cases that were graded with prior versions of CTCAE were re-graded based on the documented clinical assessment. Side effects occurring within 6 months following brachytherapy were classified as acute and those occurring after 6 months were called chronic.

\section{Intervention}

The patients were treated with high-dose-rate brachytherapy with an acrylic surface mold technique. High-dose-rate was chosen over low-dose-rate brachytherapy as the latter technique was not shown to have improved outcomes, requires the patient to be hospitalized, and results in staff exposure to radiation $[11,19]$. An acrylic mold was fabricated by our dental oncologist with the afterloading catheters inserted one $\mathrm{cm}$ apart. The number of catheters - ranging from one to five - was determined based on lesion size to encompass the tumor or postoperative cavity. The medical physicist and radiation oncologist evaluated the design of the mold. The patients were comfortable with the placement of the device. A local Lidocaine spray anesthetic was used as needed to ensure patient comfort and minimize the gag reflex. The setup was checked by the radiation oncologist and medical physicist for reproducibility. An Ir-192 source was delivered via remote afterload technology through the catheters. The treatment time varied but was typically less than two minutes. An example of a brachytherapy treatment plan is shown in Figure 1. Various radiation doses and fractionations either as a primary treatment or as a boost to EBRT were used depending on the clinical scenario. External beam radiotherapy was utilized in cases where SMB coverage was determined to be insufficient to cover the tumor or postoperative biologically effective dose. Optimal dosimetry was determined using the mold before initiation of treatment. Biologically effective dose was calculated by the formula: nd $[1+d /(\alpha / \beta)]$ [20], $\alpha / \beta$ is a ratio unique for a given tissue, $d=$ dose per fraction and $n=$ number of fractions, $\alpha / \beta$ of 10 was used for the calculations [21].

\section{Statistical analysis}

The Kaplan-Meier product estimator was used to assess LC (no recurrence in the primary site), locoregional control (LRC, no recurrence in the primary site or regional lymph nodes), distant metastasis-free survival (DMFS, survival without developing distant metastases), and overall survival (OS) from the time of radiotherapy completion. Cox proportional hazards regression analysis was used to assess the relationship of patient outcomes with the following variables: patient's treatment year (grouped into three decades - 1990s, 2000s and 2010s), total biologically effective dose (EBRT and brachytherapy when the latter was administered as a boost), Karnofsky performance status, age, tumor stage and brachytherapy modality (primary or boost). Due to the limited number of patients, all comparisons were univariate. When the hazard rate was estimated on the boundary (zero or infinity), no confidence intervals were given, and the $p$-value was determined through a deviance test of the particular predictor [9]. The same analyses were performed with the data excluding the patient with oligometastatic bladder cancer metastatic to the gingiva to confirm that the results were not substantially different when only primary oral/oropharyngeal cancer patients were included. An insufficient number of patients was enrolled to assess correlations with toxicity outcomes.

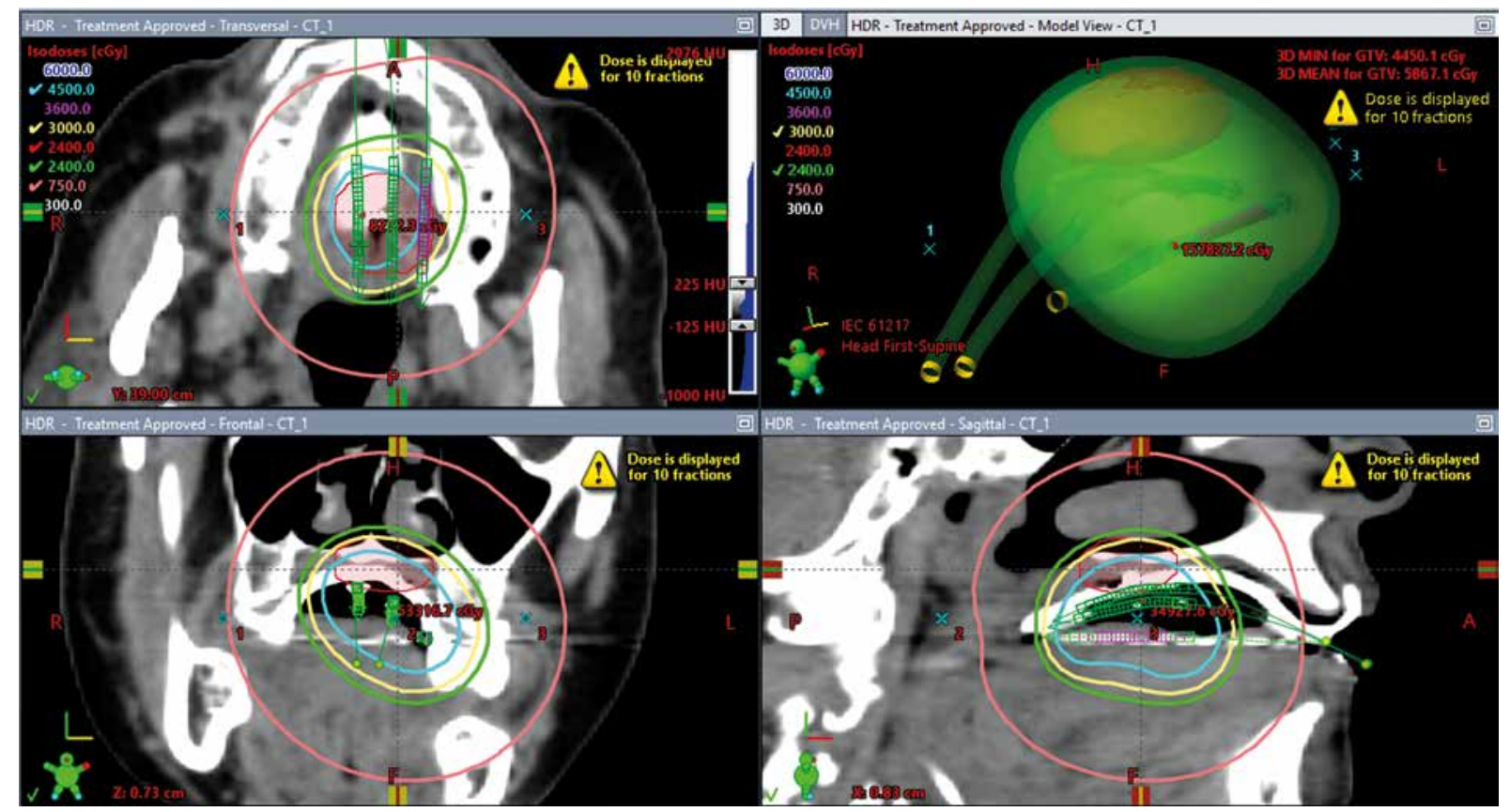

Fig. 1. Example of isodose lines of a patient treated with surface mold brachytherapy for a hard palate minor salivary gland adenocarcinoma. Isodose line color legend: cyan - 45 Gy, yellow - 30 Gy, green - 24 Gy, orange - 7.5 Gy 
Table 1. Patient characteristics

\begin{tabular}{|c|c|c|}
\hline & Number & $\%$ \\
\hline \multicolumn{3}{|l|}{ Site treated } \\
\hline Gingiva & 6 & 33 \\
\hline Soft palate & 4 & 22 \\
\hline Hard palate & 4 & 22 \\
\hline Alveolar ridge & 3 & 17 \\
\hline Tonsil & 1 & 6 \\
\hline \multicolumn{3}{|l|}{ Histology } \\
\hline Squamous cell carcinoma & 15 & 83 \\
\hline Minor salivary gland adenocarcinoma & 1 & 6 \\
\hline Melanoma & 1 & 6 \\
\hline Transitional cell carcinoma & 1 & 6 \\
\hline \multicolumn{3}{|l|}{ Stage } \\
\hline In-situ & 2 & 11 \\
\hline । & 7 & 39 \\
\hline ॥ & 7 & 39 \\
\hline IVA & 2 & 11 \\
\hline \multicolumn{3}{|l|}{ Sex } \\
\hline Male & 9 & 50 \\
\hline Female & 9 & 50 \\
\hline \multicolumn{3}{|l|}{ Age (years) } \\
\hline Mean & 63 & \\
\hline Range & $29-82$ & \\
\hline \multicolumn{3}{|l|}{ Laterality } \\
\hline Right & 9 & 50 \\
\hline Left & 6 & 33 \\
\hline Midline & 3 & 17 \\
\hline \multicolumn{3}{|l|}{ Maximum tumor dimension $(\mathrm{cm})$} \\
\hline Mean & 1.87 & \\
\hline Range & $0.2-3.5$ & \\
\hline \multicolumn{3}{|l|}{ Surgery performed prior to radiotherapy } \\
\hline Yes & 9 & 50 \\
\hline No & 9 & 50 \\
\hline \multicolumn{3}{|l|}{ Karnofsky performance status } \\
\hline 100 & 1 & 6 \\
\hline 90 & 8 & 44 \\
\hline 80 & 8 & 44 \\
\hline 70 & 1 & 6 \\
\hline \multicolumn{3}{|l|}{ Year treated } \\
\hline 2010-2018 & 3 & 17 \\
\hline 2000-2019 & 10 & 56 \\
\hline 1989-1999 & 5 & 28 \\
\hline \multicolumn{3}{|l|}{ Comorbidities } \\
\hline Smoking & 15 & 83 \\
\hline Mean pack-year history & 37 & \\
\hline Range of pack-year history & $8-160$ & \\
\hline
\end{tabular}

All analyses were performed using the R project for statistical computing software, version 3.6.2. Significance was defined as $\alpha<0.05$ [22].

\section{Results}

\section{Patient characteristics}

The detailed patient characteristics are shown in Table 1. Fifteen patients had squamous cell carcinoma histology, and the remaining patients had melanoma (1), minor salivary gland adenocarcinoma (1), and bladder transitional cell carcinoma (1). Of note, the patient with oligometastatic bladder cancer was treated for durable LC as he had no other evidence of active metastatic disease at the time of brachytherapy to the gingival metastasis. The median follow-up time was 19 months (range 3-322 months, interquartile range 12 to 66 months). At the time of brachytherapy, tumors were either completely resected $(n=9)$ or were superficial and treated with radiotherapy $(n=9)$. Indications for treatment were primary tumor (13), local recurrence (2), locoregional recurrence (1), and durable LC in a single site oligometastatic disease (1). The following sites were treated: gingiva (5), soft palate (4), hard palate (4), alveolar ridge (3), and tonsil (1).

\section{Treatment characteristics}

Treatment characteristics are shown in Table 2. 10 patients received brachytherapy alone and 8 received EBRT with a brachytherapy boost. The radiation dose for patients treated with definitive SMB was 30 Gy in 10 fractions (range 30-40 Gy in 6-12 fractions). In patients treated with brachytherapy as a boost, the median brachytherapy dose was 19 Gy in 4 fractions (range 11-30 Gy in 2-6 fractions). The median EBRT dose was 50 Gy in 25 fractions (range 30-55 Gy in 15-25 fractions). Brachytherapy was delivered twice a day (6), weekly (5), twice a week (4), or daily (3). The prescription depth ranged from surface to $1 \mathrm{~cm}$ with a median of $0.5 \mathrm{~cm}$.

\section{Treatment outcomes}

Treatment outcome details are shown in Table 3. The results of the statistical analysis are shown in Table 4. The one- and two-year LC rates were $81 \%$ and $68 \%$, LRC rates were $77 \%$ and $64 \%$, DMFS was $81 \%$ and $81 \%$, and OS was $77 \%$ and $46 \%$. The Kaplan-Meier curves are shown in Figure 2. At three years, the confidence intervals were wide but LC was 51\%, LRC 48\%, metastatic disease-free survival $81 \%$, and OS 39\%. Having stage 4 disease (relative to stage 1) was associated with inferior local and LRC and displayed a strong trend toward decreased overall survival. Age, Karnofsky performance status, decade when the patients were treated, total biologically effective dose, and whether radiotherapy was used as a boost or definitively did not correlate with the outcomes. These associations remained, and the control/survival rates were very similar when the patient with oligometastatic bladder cancer metastatic to the gingiva was excluded from the analyses. Treatment outcomes by site treated, histology, stage and radiotherapy used are shown in Table 5. 
Table 2. Treatment characteristics

\begin{tabular}{|c|c|c|}
\hline & Number & $\%$ \\
\hline \multicolumn{3}{|l|}{ Brachytherapy use } \\
\hline Brachytherapy alone & 10 & 56 \\
\hline $\begin{array}{l}\text { Brachytherapy boost to } \\
\text { external beam radiation therapy }\end{array}$ & 8 & 44 \\
\hline \multicolumn{3}{|l|}{ Treatment intent } \\
\hline Definitive & 17 & 94 \\
\hline Palliative & 1 & 6 \\
\hline \multicolumn{3}{|l|}{ Treatment timing } \\
\hline Initial treatment & 16 & 89 \\
\hline Recurrent disease & 2 & 11 \\
\hline \multicolumn{3}{|l|}{ Brachytherapy dose (Gy) } \\
\hline Mean (Gy) & 27 & \\
\hline Range (Gy) & $11-36$ & \\
\hline Mean for brachytherapy alone (Gy) & 33 & \\
\hline Mean for brachytherapy boost (Gy) & 19 & \\
\hline \multicolumn{3}{|l|}{ Brachytherapy number of fractions } \\
\hline Mean & 7 & \\
\hline Range & $2-15$ & \\
\hline \multicolumn{3}{|l|}{ Brachytherapy frequency } \\
\hline Twice a day & 6 & \\
\hline Once a week & 5 & \\
\hline Twice a week & 4 & \\
\hline Daily & 3 & \\
\hline \multicolumn{3}{|l|}{$\begin{array}{l}\text { External beam radiation dose prior to } \\
\text { brachytherapy when used }\end{array}$} \\
\hline Mean (Gy) & 48 & \\
\hline Range (Gy) & $30-55$ & \\
\hline \multicolumn{3}{|l|}{$\begin{array}{l}\text { External beam radiation number } \\
\text { of fractions prior to brachytherapy } \\
\text { when used }\end{array}$} \\
\hline Mean & 23 & \\
\hline Range & $15-25$ & \\
\hline \multicolumn{3}{|l|}{ Total biologically effective dose } \\
\hline Mean & 64 & \\
\hline Range & $36-115$ & \\
\hline \multicolumn{3}{|l|}{$\begin{array}{l}\text { Brachytherapy computed tomography } \\
\text { simulation performed }\end{array}$} \\
\hline Yes & 1 & 6 \\
\hline No & 17 & 94 \\
\hline \multicolumn{3}{|l|}{ Brachytherapy prescription depth (cm) } \\
\hline Mean & 0.6 & \\
\hline Range & $0-1$ & \\
\hline \multicolumn{3}{|l|}{ Number of brachytherapy catheters used } \\
\hline Mean & 3 & \\
\hline Range & $1-5$ & \\
\hline
\end{tabular}

\section{Toxicity}

The description of radiation-induced side effects can be found in Table 3. Due to the low number of patients, statistical analyses could not be performed to assess the factors
Table 3. Treatment outcomes

\begin{tabular}{|c|c|c|}
\hline & Number & $\%$ \\
\hline \multicolumn{3}{|c|}{ Time since treatment completion (months) } \\
\hline Mean & 63 & \\
\hline Range & $3-322$ & \\
\hline \multicolumn{3}{|l|}{ Response to brachytherapy } \\
\hline Complete response & 9 & 50 \\
\hline Local recurrence & 4 & 22 \\
\hline Distant metastases & 3 & 17 \\
\hline Local and regional recurrence & 1 & 6 \\
\hline \multicolumn{3}{|l|}{ Time to recurrence (months) } \\
\hline Mean & 11 & \\
\hline Range & $2-28$ & \\
\hline \multicolumn{3}{|l|}{ Acute toxicity } \\
\hline Mucositis & 15 & \\
\hline Dysesthesia & 1 & \\
\hline Odynophagia & 2 & \\
\hline Dysphagia & 1 & \\
\hline Dermatitis & 2 & \\
\hline Bleeding & 1 & \\
\hline Infection & 1 & \\
\hline Fatigue & 1 & \\
\hline Xerostomia & 3 & \\
\hline \multicolumn{3}{|c|}{ Acute toxicity grade per CTCAE version 5.0} \\
\hline None & 1 & 6 \\
\hline Grade 1 & 8 & 44 \\
\hline Grade 2 & 8 & 44 \\
\hline Grade 3 & 1 & 6 \\
\hline \multicolumn{3}{|c|}{ Late toxicity grade per CTCAE version 5.0} \\
\hline None & 14 & 78 \\
\hline Grade 1 & 3 & 17 \\
\hline Xerostomia & 1 & 6 \\
\hline Telangiectasia & 1 & 6 \\
\hline Fibrosis & 1 & 6 \\
\hline Trismus & 1 & 6 \\
\hline Wound healing complications & 1 & 6 \\
\hline Grade 2 & 1 & 6 \\
\hline Mandible osteoradionecrosis & 1 & 6 \\
\hline
\end{tabular}

that affect these toxicities. The acute toxicity outcomes were very favorable: no toxicity in one patient, grade 1 in seven cases, grade 2 in nine, and grade 3 in one patient. The most common toxicity was mucositis, which occurred in $83 \%$ of patients. Xerostomia occurred in three and odynophagia in two cases. Late effects were rare, only occurring in three patients. These included grade 1 fibrosis and trismus, xerostomia, wound complications; and grade 2 osteoradionecrosis of the mandible. The latter developed in a patient who had multiple major comorbidities including a 160-packyear history of smoking, four prior primary malignancies 


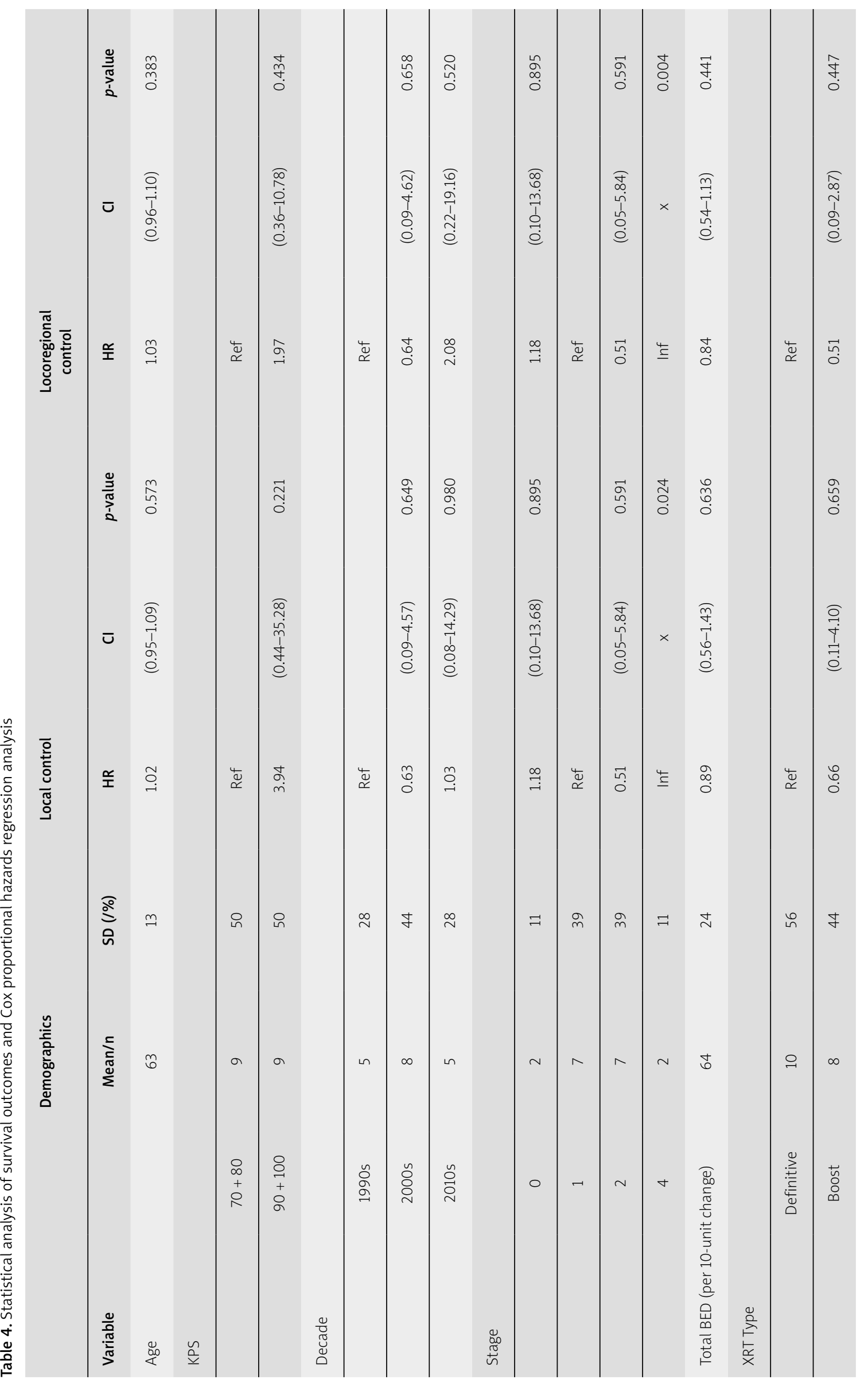




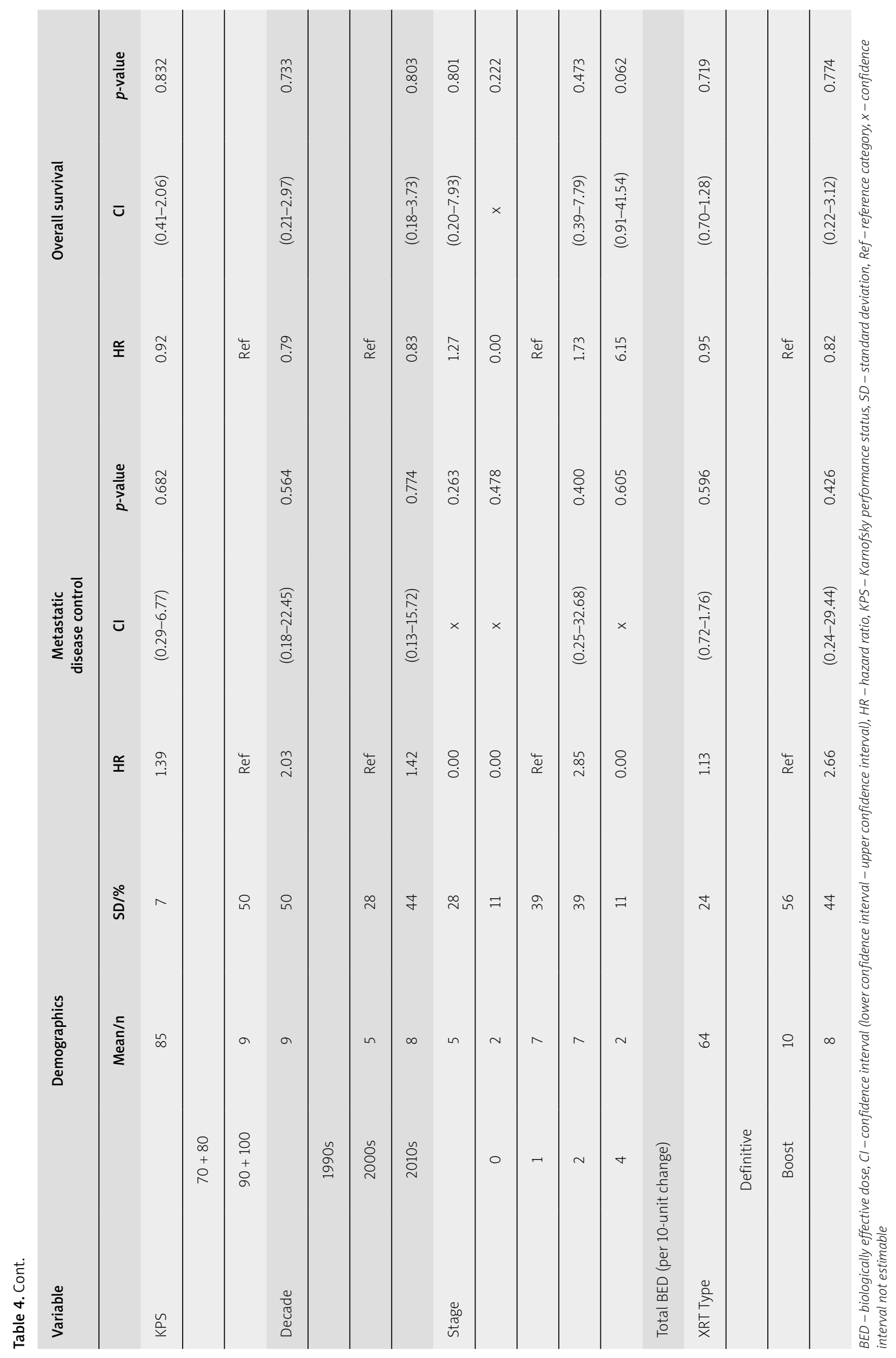



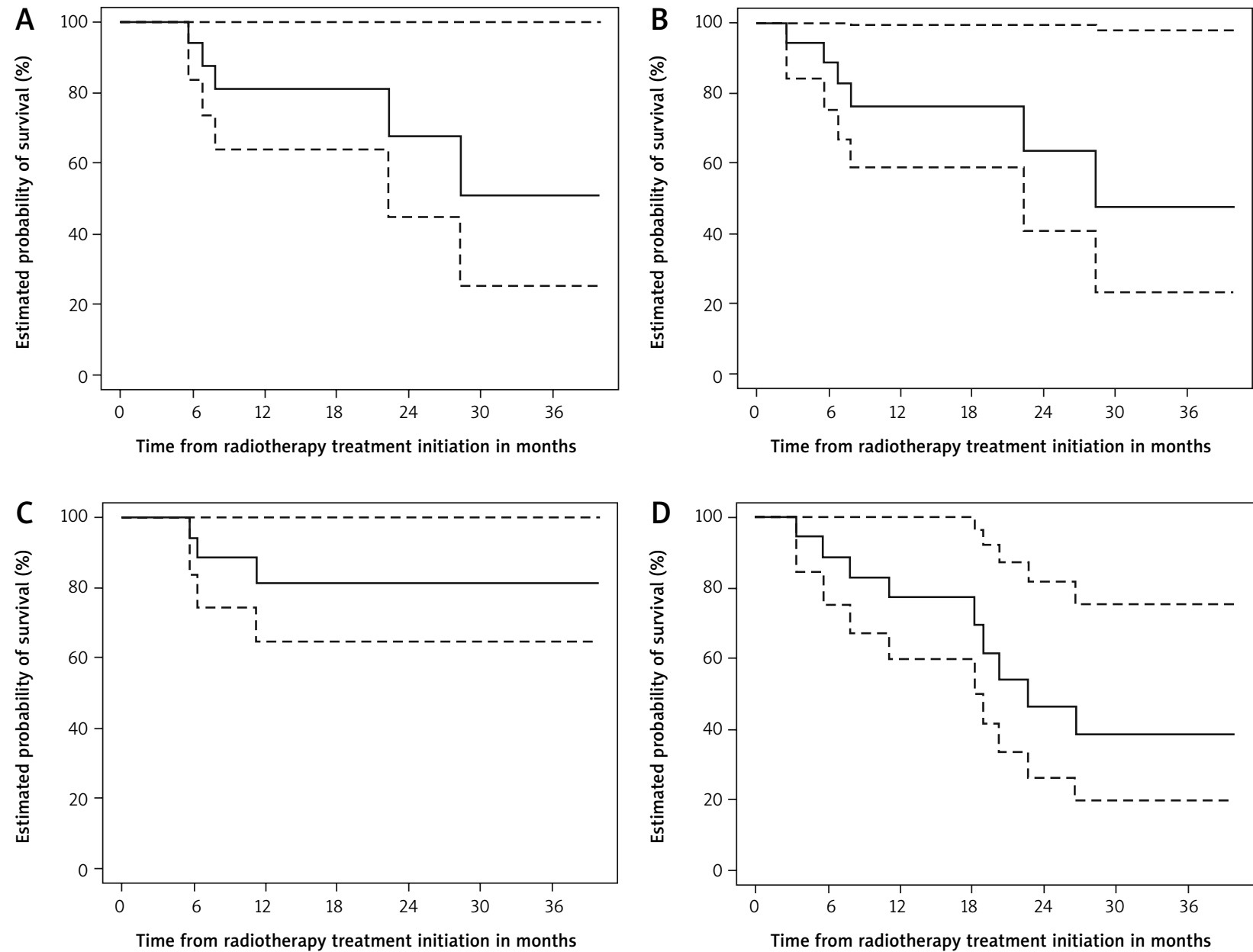

Fig. 2. Kaplan-Meier curves of disease control: (A) local control, (B) locoregional control, (C) metastatic disease control, (D) overall survival

including floor of mouth and base of the tongue squamous cell carcinomas, hypertension, and deep venous thrombosis.

\section{Discussion}

We report our institutional experience with SMB in superficial oral and oropharyngeal cancers. The goal was to evaluate the safety and efficacy of this modality. Disease-specific outcomes and toxicity were evaluated, and clinical factors associated with these were analyzed.

\section{Treatment outcomes}

In our report, the 2 -year LC is $68 \%$. The 3 -year $\mathrm{LC}$ rate is $51 \%$, but the confidence interval ranged from $25 \%$ to $100 \%$. While the statistical power was low due to the small number of patients, stage 4 disease was associated with inferior local and locoregional tumor control. This suggests that these patients may not be ideal candidates for the SMB approach. Our results were similar even when the oligometastatic bladder cancer patient with a gingival metastasis was excluded from the analysis.

In prior single-institutional retrospective studies, LC rates with the surface mold technique range from 50 to $100 \%$ depending on the exact technique used, tumor location, and thickness $[2,6-8,14,15,17,23-26]$. No prospective data are available. All the studies on SMB in oral cancers were conducted in Asia or India. In the largest study of SMB, by Takeda et al. in 27 patients, brachytherapy alone in oral cavity tumors with a thickness of fewer than $2 \mathrm{~mm}$ and a combination of EBRT and brachytherapy in thicker tumors were used. Local control was 74\% at five years [24]. Budrukkar et al. reported 21 patients with oral/oropharyngeal cancers treated with SMB and reported a 5 -year LC of $76 \%$ for intra-oral malignancies with tumor control in an initial treatment setting to salvage radiotherapy of recurrent disease [15]. Unetsubo et al. in a report of 17 patients found a 5 -year LC of $54 \%$, but when patients with floor of the mouth and posterior region of the buccal mucosa tumors were excluded, the 5-year LC was 72\% [8]. Ealla et al. observed no recurrences in six patients with T1NOMO hard palate cancers treated with SMB [27]. Murkherji et al. reported that all of their nine patients achieved LC with only one nodal recurrence [2]. In buccal mucosa and lip, customized mold brachytherapy techniques utilized in five patients also achieved tumor control in all cases, as demonstrated by Matsuzaki et al. [6]. Likewise, Chatani et al. found no tumor recurrence in all nine treated patients with oral cavity cancers [17]. Ariji et al. reported another study that showed tumor control in all patients in a small sample of four patients [14]. In a two-patient report of maxillary gingival carcinomas treated with SMB by Kudoh et al., no recurrences were noted [7]. Nishimura 


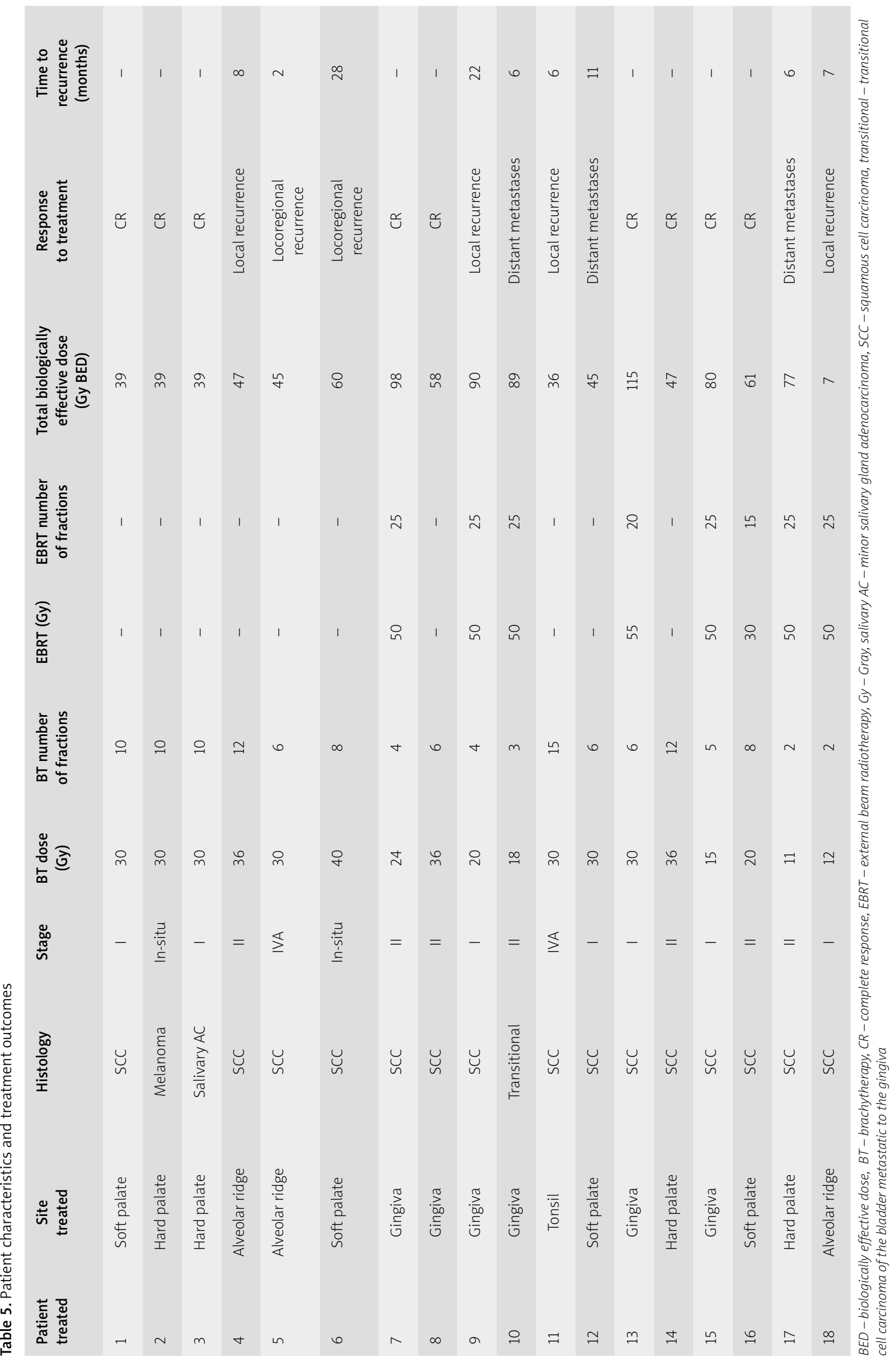


et al., however, recorded a poor LC of $50 \%$ in eight patients. This was hypothesized to be due to the inclusion of tumors located in the retromolar trigone and the possibility of including tumors with greater depth of invasion than $5 \mathrm{~mm}$ [23].

In the literature, a wide range of doses and fractionations has been used in SMB, from 12 Gy in 2 fractions to $52.5 \mathrm{~Gy}$ in 15 fractions either daily, twice daily or two to three times a week. Brachytherapy has also been reported as a sole modality and as a boost to EBRT $[2,6-8,14,15$, 17, 23-25]. In our patients, superficial brachytherapy was chosen in very superficial cancers or a lower-risk adjuvant setting. It was also used as a boost to EBRT when feasible. The doses and fractionations used in our patients were consistent with the published studies as shown in Table 2.

\section{Toxicity}

In our study, acute side effects were noted in all but one patient. These, as expected, were mainly grades 1-2 with only one case of grade 3 toxicity. The low toxicity profile that we are reporting is consistent with many of the prior studies of SMB. Mukherji et al. noted grade 1-2 radiation mucositis acutely in all patients and some late superficial skin hypopigmentation in half of all cases but no serious late toxicity such as ulcers, necrosis, or strictures [2]. Likewise, no serious late complications were noted by Nishimura et al. [23]. Budrukkar et al. reported 17\% grade 2 skin and 31\% grade 2 mucosal toxicity. More concerning, one case of palatal perforation of the soft palate and one bone exposure were noted [15]. Ariji et al. reported no major side effects from radiotherapy [14]. Unetsubo et al., Ealla et al. and Matsuzaki et al. noted a 100\% rate of acute grade $1-2$ radiation mucositis as well as some cases of radiation dermatitis $[6,8,27]$. Unetsubo et al. noted one case of grade 3 palatal mucosal ulceration in a patient with a hard palate primary and one case of grade 3 fistula between the skin and buccal mucosa in a buccal mucosal primary [8]. However, some studies had concerning findings. In the largest study of SMB, by Takeda et al., more than half of treated patients developed bone exposure 5 months to 3 years after brachytherapy, two of whom required surgical intervention for osteoradionecrosis [24]. Also, in both treated patients with gingival carcinoma, necrosis, ulcer formation, and bone exposure were noted by Kudoh et al. [7]. The prior studies did not evaluate whether patients' comorbidities played a role in the development of radiation-induced late effects. In our study, the patients who developed chronic side effects were all smokers with multiple serious medical comorbidities. The patient who developed grade 2 mandible osteoradionecrosis had a particularly extensive history of prior malignancies, smoking, and cardiovascular disease.

\section{Limitations}

This study provides important data on the treatment of oral and oropharyngeal lesions where there is a paucity of prospective studies and there is no consensus among experts. However, our sample size is relatively small and included a heterogeneous group of patients. Most patients treated at our institution received external beam radiation therapy. Subgroup analyses were infeasible due to the limited number of patients. The study spans thirty years, which is a limitation since treatment practices and tumor staging changed over this period. Our study is retrospective, which limits our ability to determine how much of the tumor control, toxicity, and survival benefit was specifically due to brachytherapy. Another limitation is the variation in systemic therapy; surgical and radiotherapy regimens were utilized in our patients before and after brachytherapy.

\section{Future directions}

Prospective studies comparing SMB with the other radiotherapy techniques are needed to further clarify the safety and effectiveness of this modality. Unfortunately, to the best of our knowledge, no such studies have been conducted or are ongoing. Larger retrospective studies - ideally multi-institutional - should clarify which patients are best selected for this approach. It appears that elderly and surgically inoperable patients could be offered SMB in select cases. However, the response of tumors in various sites of the oral cavity such as the floor of the mouth, retromolar trigone, buccal mucosa, gum, and palate to different radiation doses and fractionations needs to be evaluated further.

\section{Acknowledgment}

The study was approved through the Institutional Review Board review by our university.

\section{Conclusions}

Surface mold brachytherapy is a viable modality as either primary or boost treatment for superficial oral cancers. This treatment method has a low toxicity profile and offers reasonable tumor control in select patients. Stage 4 disease patients may have inferior local and LRC with SMB.

The authors declare no conflict of interest.

\section{References}

1. Mourad M, Jetmore $T$, Jategaonkar AA, et al. Epidemiological trends of head and neck cancer in the United States: a SEER population study. J Oral Maxillofac Surg 2017; 75: 2562-2572.

2. Mukherji A, Mourougan S, Saravannan K, et al. Dosimetric analysis and clinical outcomes in CT-based mould brachytherapy in early oral cancers in patients unfit for surgery. J Contemp Brachytherapy $2015 ; 7:$ 147-153.

3. Garran C, Montesdeoca N, Martinez-Monge R. Treatment of upper gum carcinoma with high-dose-rate customized-mold brachytherapy. Brachytherapy 2008; 7: 267-269.

4. Rosenthal DI, Mohamed ASR, Garden AS, et al. Final report of a prospective randomized trial to evaluate the dose-response relationship for postoperative radiation therapy and pathologic risk groups in patients with head and neck cancer. Int J Radiat Oncol Biol Phys 2017; 98: 1002-1011.

5. Main J, Orr J, McGurk F, et al. Salivary gland tumors: review of 643 cases. J Oral Pathol 1976; 5: 88-102.

6. Matsuzaki H, Takemoto M, Hara M, et al. Two-piece customized mold technique for high-dose-rate brachytherapy on cancers of the buccal mucosa and lip. Oral Surg Oral Med Oral Pathol Oral Radiol 2012; 113: 118-125.

7. Kudoh T, Ikushima H, Kudoh K, et al. High-dose-rate brachytherapy for patients with maxillary gingival carcinoma using a novel cus- 
tomized intraoral mold technique. Oral Surg Oral Med Oral Pathol Oral Radiol Endod 2010; 109: e102-108.

8. Unetsubo T, Matsuzaki H, Takemoto M, et al. High-dose-rate brachytherapy using molds for lip and oral cavity tumors. Radiat Oncol 2015; 10: 81.

9. Silva SR, Martin B, Choi M, et al. National Cancer Database Analysis of the effect of brachytherapy on overall survival in patients with base of tongue cancer. Head Neck 2019; 41: 1184-1192.

10. Nose T, Koizumi M, Nishiyama K. High-dose-rate interstitial brachytherapy for oropharyngeal carcinoma: results of 83 lesions in 82 patients. Int J Radiat Oncol Biol Phys 2004; 59: 983-991.

11. Kovacs G, Martinez-Monge R, Budrukkar A, et al. GEC-ESTRO ACROP recommendations for head \& neck brachytherapy in squamous cell carcinomas: $1^{\text {st }}$ update - improvement by cross sectional imaging based treatment planning and stepping source technology. Radiother Oncol 2017; 122: 248-254.

12. Petera J, Sirak I, Laco J, et al. High-dose-rate brachytherapy in early oral cancer with close or positive margins. Brachytherapy 2015; 14: 77-83.

13. Wu WJ, Shao X, Huang MW, et al. Postoperative iodine-125 interstitial brachytherapy for the early stages of minor salivary gland carcinomas of the lip and buccal mucosa with positive or close margins. Head Neck 2017; 39: 572-577.

14. Ariji E, Hayashi N, Kimura Y, et al. Customized mold brachytherapy for oral carcinomas through use of high-dose-rate remote afterloading apparatus. Oral Surg Oral Med Oral Pathol Oral Radiol Endod 1999; 87: 508-512.

15. Budrukkar A, Dasgupta A, Pandit P, et al. Clinical outcomes with high-dose-rate surface mould brachytherapy for intra-oral and skin malignancies involving head and neck region. J Contemp Brachytherapy 2017; 9: 242-250.

16. Cengiz M, Ozyar E, Ersu B, et al. High-dose-rate mold brachytherapy of early gingival carcinoma: a clinical report. J Prosthet Dent 1999; 82: 512-514.

17. Chatani M, Tsuboi K, Yagi M, et al. High-dose-rate brachytherapy using molds after chemoradiotherapy for oral cavity cancer. Jpn J Radiol 2012; 30: 40-44.

18. Common Terminology Criteria for Adverse Events (CTCAE) Version 5.0

19. Inoue T, Inoue T, Teshima T, et al. Phase III trial of high and low dose rate interstitial radiotherapy for early oral tongue cancer. Int J Radiat Oncol Biol Phys 1996; 36: 1201-1204.

20. Jones B, Dale RG, Deehan C, et al. The role of biologically effective dose (BED) in clinical oncology. Clin Oncol (R Coll Radiol) 2001; 13: 71-81.

21. Stuschke M, Thames HD. Fractionation sensitivities and dose-control relations of head and neck carcinomas: analysis of the randomized hyperfractionation trials. Radiother Oncol 1999; 51: 113-121.

22. Klein JPM, M.L. Survival Analysis Techniques for Censored and Truncated Data. Statistics for Biology and Health 2003.

23. Nishimura Y, Yokoe Y, Nagata, Y, Okajima K, Nishida M, Hiraoka M. High-dose-rate brachytherapy using molds for oral cavity cancer. The technique and its limitations. Int I Clin Oncol 1998; 3: 351-356.

24. Takeda M, Shibuya H, Inoue T. The efficacy of gold-198 grain mold therapy for mucosal carcinomas of the oral cavity. Acta Oncol 1996; 35: 463-467.

25. Yoden E, Hiratsuka J, Imajo Y, et al. High-dose-rate microselectron mould ratiotherapy of a widespread superficial oral cancer. Int J Oral Maxillofac Surg 1999; 28: 451-453.

26. Reshko L, Khan Z, Sowards KT, et al. Squamous cell and adenoid cystic carcinoma collision tumor of the soft palate treated with surface mold brachytherapy. Cureus 2020; 12: e7297.

27. Ealla KKR, Prakash SKC, Manidanappanavar P, et al. Treatment of stage I hard palate cancer with high-dose-rate brachytherapy using molds: cost-based perspective. J Pharm Bioallied Sci 2019; 11: S67-s71.

\section{Address for correspondence}

Leonid Borisovich Reshko, MD

University of Louisville, the United States of America

e-mail: Ibresh01@louisville.edu

Submitted: 05.10.2021

Accepted: 31.10 .2021 